\title{
Thoracoscopic lobectomy: Evolving and improving
}

Thomas A. D'Amico, MD

See related article on page 507 .

From the Department of Surgery, Duke University Medical Center, Durham, NC.

Received for publication April 19, 2006; accepted for publication April 24, 2006.

Address for reprints: Thom A. D'Amico, MD, Associate Professor of Surgery, Duke University Medical Center, Box 3496, Durham, NC 27710 (E-mail: damic001@ mc.duke.edu).

J Thorac Cardiovasc Surg 2006;132:464-5

$0022-5223 / \$ 32.00$

Copyright (๑) 2006 by The American Association for Thoracic Surgery

doi:10.1016/j.jtcvs.2006.04.026
$\mathrm{T}$ horacoscopic lobectomy, also termed video-assisted thoracoscopic (VATS) lobectomy, has been demonstrated to be a safe and effective procedure to treat early-stage non-small cell lung cancer (NSCLC). Advantages of thoracoscopic lobectomy, as compared with lobectomy with thoracotomy, include less postoperative pain, faster return to full activity, preserved pulmonary function, shorter chest tube duration and length of hospitalization, reduced inflammatory response, and a lower rate of postoperative atrial fibrillation. ${ }^{1,2}$

Despite these advantages, thoracoscopic lobectomy has not achieved the expected widespread acceptance seen with other minimally invasive procedures. One important obstacle to the development of this procedure has been confusion regarding the technical aspects of the procedure - the attachment of the term "VATS lobectomy" to a number of procedures with varying degrees of invasiveness and oncologic effectiveness. Thoracoscopic lobectomy should be defined as a completely thoracoscopic procedure performed with a limited number of ports (one or two) and an access incision (approximately $5 \mathrm{~cm}$ in length) for individual vessel and bronchial dissection and ligation (stapling), as well as specimen removal. ${ }^{3}$ Importantly, rib spreading with retractors is not used. "Video-assisted" procedures that include thoracotomy with rib spreading or rib resection do not qualify, because the advantages of the minimally invasive approach are attributed to the avoidance of this practice.

Another obstacle has been the uncertainty regarding oncologic effectiveness of the thoracoscopic approach. Although there are no prospective, randomized studies comparing survival after the open and thoracoscopic approaches, there is also no evidence to support a difference if the procedure is performed as described. ${ }^{3}$ Large series describing extensive experience with thoracoscopic lobectomy report stagespecific survival that is at least equivalent to historical series. ${ }^{2}$ Furthermore, concerns regarding the effectiveness of the thoracoscopic mediastinal lymph node dissection are unfounded. This has been demonstrated as well. ${ }^{1-4}$

The study by Shigemura and colleagues ${ }^{4}$ analyzes surgical outcomes for clinical stage IA NSCLC (tumor size $\leq 2 \mathrm{~cm}$ only), comparing completely thoracoscopic, VATS-assisted, and open techniques. The premise of the study is that there is a lack of studies with "long-term follow-up" in comparing VATS with alternative techniques. This is not entirely true, as several centers have extensive experience and reasonable follow-up. ${ }^{1,2,5}$ Furthermore, the premise that there is controversy regarding the 3 procedures studied is misleading. The advantages associated with completely thoracoscopic lobectomy are attributable to the avoidance of rib spreading, and the use of VATS "assistance" during minithoracotomy does not confer these advantages.

This study is difficult to interpret because several of the primary outcomes differ from what would be found in other studies. One example is length of hospitalization: in this study, hospital stay is shorter in the thoracoscopic group than the other 2 groups, a finding consistent with the literature. However, the result is 4 times longer than in other studies (12 days vs 3 days). ${ }^{1,2}$ Thus, the conclusion that the completely thoracoscopic technique is associated with a shorter length of stay than the alternatives is not clinically relevant. In addition, the operative times and survival are much longer than in other studies, and these deviations go unexplained.

Of note, this study is the first to document statistically that there is no difference in the ability to perform a complete mediastinal lymph node dissection by enumerating the lymph nodes removed. The thoracoscopic approach included an equivalent 
number of lymph nodes $(23 \pm 9)$ as compared with the open approach $(25 \pm 7 ; P=$ not significant $) .{ }^{4}$ Other questions regarding this study arise. It is unclear why the authors chose $2 \mathrm{~cm}$ as the cutoff for this study of clinical stage IA lung cancer. It is also unclear how patients were chosen for 1 of the 3 operations. Is there any selection bias? The study of 145 patients is not powered to show anything but a huge difference in survival, which would not be expected. Thus, survival is not a meaningful end point for comparison in this study. In summary, this study confirms that thoracoscopic lobectomy is a safe and effective approach for the resection of early-stage NSCLC and that mediastinal lymph node dissection is not compromised by the thoracoscopic approach.

With increasing experience, the indications for thoracoscopic lobectomy have been expanded. Thoracoscopic lobectomy has been found to be applicable to larger stage I and II tumors, central pulmonary metastases, ${ }^{2,5}$ granulomatous lung disease, and to patients after induction chemotherapy. ${ }^{5}$ Thoracic surgery training programs may soon be required to demonstrate faculty competency in thoracoscopic lobectomy to improve resident experience. Although specific training for this procedure has been successful in dedicated courses and preceptorships for surgeons in practice, providing this training during residency would be ideal.

Confirmation of the oncologic effectiveness of thoracoscopic lobectomy would be best demonstrated by a large, prospective, randomized series, which will not be forthcoming. A phase III trial comparing thoracoscopic to conventional lobectomy would require accrual of more than 800 patients, which is prohibitive. Furthermore, there is not sufficient equipoise among most thoracoscopic surgeons to permit randomization. However, a prospective registry trial has been proposed by the Cancer and Leukemia Group B (CALGB) and will likely be approved and funded. The CALGB trial will accept accrual of lobectomy patients, using either the completely thoracoscopic approach or thoracotomy, comparing survival and quality of life. Although not randomized, the registry design will allow comparisons of important variables in appropriately matched patients. Finally, the role of thoracoscopic lobectomy is currently being explored in patients receiving adjuvant chemotherapy. It is possible that the thoracoscopic approach will improve patient compliance with chemotherapy and ultimately improve survival.

\section{References}

1. Daniels LJ, Balderson SS, Onaitis MW, D’Amico TA. Thoracoscopic lobectomy: a safe and effective strategy for patients with stage I lung cancer. Ann Thorac Surg. 2002;74:860-4.

2. McKenna RJ, Houck W, Fuller CB. Video-assisted thoracic surgery lobectomy: experience with 1,100 cases. Ann Thorac Surg. 2006;81: 421-6.

3. Burfeind WR, D'Amico TA. Thoracoscopic lobectomy. Op Tech Thorac Cardiovasc Surg. 2004;9:98-114.

4. Shigemura N, Akashi A, Funaki S, Nakagiri T, Inoue M, Sawabata N, et al. Long-term outcomes after a variety of video-assisted thoracoscopic lobectomy approaches for clinical stage IA lung cancer: A multi-institutional study. J Thorac Cardiovasc Surg. 2006;132:507-12.

5. Petersen RP, Pham DK, Toloza EM, Burfeind WR, Harpole DH, Hanish SI, et al. Thoracoscopic lobectomy: a safe and effective strategy for patients receiving induction therapy for non-small cell lung cancer. Ann Thorac Surg. 2006;82:214-9. 